\title{
ON A THEORY FOR UNSTEADY MOTION OF RECTANGULAR WING IN SUPERSONIC FLOW*
}

\author{
By \\ MILOMIR M. STANIŠIC \\ Purdue University
}

1. Introduction. The problem of the determination of the lifting forces acting on a wing of rectangular form in a supersonic, unsteady flow has been of interest for many years. The solution** for the motion of the wing of rectangular form in a supersonic, steady flow has been obtained by Busemann [1], Galin [2], Falkovich [3] and many others. The unsteady wing theory for the case of a wing of delta form has been developed by Miles [4], [5] and also independently by Stanišić [6], [7]. Moreover, Krassilchtchikova [8] has developed an unsteady wing theory for an arbitrary plan form of the wing by means of an integral equation which has not been solved. This integral equation is a direct consequence of boundary conditions imposed upon the lifting surface by means of the Volterra-Green's Method. The difficultiess of solving such an integral equation [9] lies in its domain of integration. For the case of the wing of delta form in [7], an approximate method to solve the corresponding integral equation has been presented.

However, if the form of the wing is rectangular, then Volterra-Green's method imposes enormous difficulties due to edge effects. Recently, Kovaleva [10] extended the technique developed by Galin for steady motion to the solution for the unsteady case. This solution is restricted by the assumption that the down-wash distribution over the lifting surface is a monotonically increasing function depending on time only. This assumption differs from the conditions appearing in practice since the actual conditions holding on the lifting surface are functions of both time and space coordinates. In this paper a new method is developed for determining the lifting forces acting on a wing of rectangular plan form subjected to the more generalized boundary conditions occurring during flight. The new method is based on technique previously used by Galin and Kovaleva. The method consists basically of two steps: Firstly, to determine the steady loading function which satisfies the more generalized boundary conditions and secondly, by using the technique of operational calculus to find the relation between steady and unsteady loading function which will yield the solution of the problem in closed form. The same method has been applied previously by the author for the solution of the problem of the unsteady motion of a delta wing in a supersonic flow with supersonic leading edges [11].

2. Formulation. Consider a rectangular wing of plain form of span $l$, and width $h$, placed in a coordinate system as shown in Fig. 1. The partial differential equation describing the disturbance of the flow in presence of a solid body is given by:

$$
-\left(M^{2}-1\right) \frac{\partial^{2} \Phi}{\partial x^{2}}+\frac{\partial^{2} \Phi}{\partial y^{2}}+\frac{\partial^{2} \Phi}{\partial z^{2}}-\frac{1}{a^{2}}\left[2 U \frac{\partial^{2} \Phi}{\partial x \partial t}+\frac{\partial^{2} \Phi}{\partial t^{2}}\right]=0
$$

\footnotetext{
*Received September 8, 1961; revised manuscript received December 18, 1961.

**The numbers in the squared brackets refer to the bibliography at the end of this paper.
} 


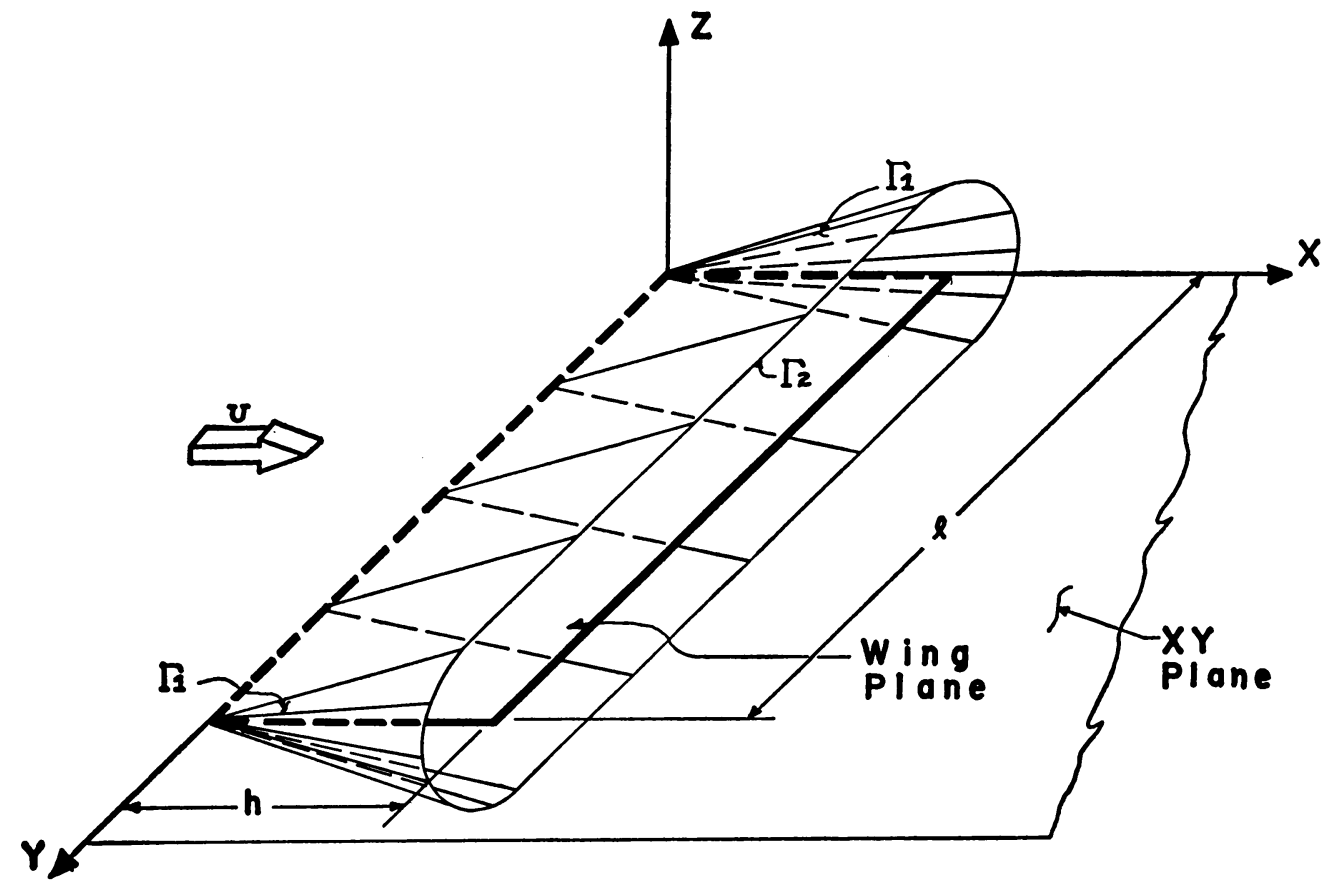

Fig. 1 Geometry of Rectangular Wing in Supersonic Flow

where $U$ is velocity of the wing, $a$ is velocity of the sound and $\Phi=\Phi(x, y, z, t)$ is the velocity potential function. Then the lifting force acting on the wing is given by:

$$
Q=-2 \rho \int_{0}^{h} \int_{0}^{l}\left(\frac{\partial \Phi}{\partial t}+U \frac{\partial \Phi}{\partial x}\right) d x d y
$$

where $\rho$ is the undisturbed density. Equation (1) is subjected to the following boundary conditions.

a) The normal component of the velocity of a wing must be equal to the normal component of the velocity of the flow, i.e.,

$$
\boldsymbol{\Omega} \cdot \mathbf{n}=\mathbf{c} \cdot \mathbf{n}
$$

where $\Omega$ is velocity of the wing, $c$ is velocity of the flow field and $n$ is normal to the lifting surface, [6]. Denote the position of the wing at any time as

$$
z=g(x, y) e^{-i \omega t}
$$

where $\omega$ is the frequency of the vibrating wing, and $g(x, y)=g$ is a prescribed function, regular every where in the region under consideration. It is easy to show [6] that the condition (3) can be written according to the linearized theory as

$$
\left.\frac{\partial \Phi}{\partial z}\right|_{z=0}=e^{-i \omega t}\left[U \frac{\partial g}{\partial x}-i \omega g\right], \quad 0 \leq x \leq h, \quad l \geq y \geq 0
$$

b) The other boundary conditions result, as stated by Kovaleva [10] from the fact that

$$
\Phi\left(\Gamma_{1}, \Gamma_{2}\right)=0, \quad \text { and } \frac{\partial}{\partial \nu} \Phi\left(\Gamma_{1}, \Gamma_{2}\right)=0
$$


where $\Gamma_{1}$ and $\Gamma_{2}$ are the characteristic surfaces [6] of the hyperbolic equation (1), and $\nu$ is conormal on the characteristic surfaces. Then, by standard substitution,

$$
\Phi(x, y, z, t)=e^{k x-i \omega t} \Psi(x, y, z) .
$$

Equation (1) can be written as

$$
-\left(M^{2}-1\right) \frac{\partial^{2} \Psi}{\partial x^{2}}+\frac{\partial^{2} \Psi}{\partial y^{2}}+\frac{\partial^{2} \Psi}{\partial z^{2}}+\lambda^{2} \Psi=0,
$$

where $\Psi=\Psi(x, y, z)$ and

$$
k=\frac{i \omega M}{a\left(M^{2}-1\right)} ; \quad \lambda^{2}=-\left(\frac{\omega}{a}\right)^{2}\left(M^{2}-1\right)^{-1} .
$$

However, Eqs. (5), (6), and (7), lead to

$$
\begin{gathered}
\left.\frac{\partial \Psi}{\partial z}\right|_{z=0}=e^{-k x}\left[U \frac{\partial g}{\partial x}-i \omega g\right], \quad 0 \leq x \leq h, \quad l \geq y \geq 0 \\
\Psi\left(\Gamma_{1}, \Gamma_{2}\right)=0 ; \quad \frac{\partial}{\partial \nu} \Psi\left(\Gamma_{1}, \Gamma_{2}\right)=0 .
\end{gathered}
$$

Equation (2) now becomes

$$
Q=-2 \rho U e^{-i \omega t} \int_{0}^{h} \int_{0}^{l}\left[\left(k-\frac{i \omega}{U}\right) \Psi+\frac{\partial \Psi}{\partial x}\right] e^{k x} d x d y .
$$

Note that (10) can be written as

$$
\left.\frac{\partial \Psi}{\partial z}\right|_{z=0}=e^{-k x} f(x, y)
$$

where

$$
f(x, y)=U \frac{\partial g}{\partial x}-i \omega g .
$$

Equation (14) can be expanded in Taylor series, namely

$$
f(x, y)=\sum_{s=0}^{\infty} \sum_{r=0}^{\infty} a_{s r} x^{s} y^{r}, \quad s=0,1,2, \cdots ; \quad r=0,1,2, \cdots,
$$

where $a_{s r}$ are coefficients given by

$$
a_{s r}=\frac{1}{s ! r !} \frac{\partial^{s} f}{\partial x^{s}} \frac{\partial^{r} f}{\partial y^{r}} \mid, \quad x=0, \quad y=0
$$

Denote

$$
x_{1}=\Lambda x, \quad y_{1}=\Lambda \mu y, \quad z_{1}=\Lambda \mu z,
$$

where

$$
\Lambda^{2}=\left(\frac{\lambda}{\mu}\right)^{2}, \quad \mu^{2}=\left(M^{2}-1\right) .
$$

Equations (8), (10), and (11) can then be written as

$$
-\frac{\partial^{2} \Psi}{\partial x_{1}^{2}}+\frac{\partial^{2} \Psi}{\partial y_{1}^{2}}+\frac{\partial^{2} \Psi}{\partial z_{1}^{2}}+\Psi=0,
$$




$$
\begin{gathered}
\left.\frac{\partial \Psi}{\partial z_{1}}\right|_{z_{1}=0}=\frac{e^{-\beta x_{2}}}{\Lambda \mu} f\left(x_{1}, y_{1}\right), \quad 0 \leq x_{1} \leq \Lambda h, \quad \Lambda \mu l \geq y \geq 0 \\
\Psi\left(\Gamma_{1}, \Gamma_{2}\right)=0 ; \quad \frac{\partial}{\partial \nu} \Psi\left(\Gamma_{1}, \Gamma_{2}\right)=0
\end{gathered}
$$

with

$$
\beta=k / \Lambda \text {. }
$$

Equations (19), (20), and (21) represent the boundary value problem under consideration.

3. Method of solution. As soon as a function $\Psi\left(x_{1}, y_{1}, z_{1}\right)$ is found such that satisfies (19) subject to the conditions (20) and (21), the problem is solved, since (12) can then be evaluated without any difficulty. The solution, $\Psi\left(x_{1}, y_{1}, z_{1}\right)$ of (19) will hereafter be called the unsteady loading function.

Moreover, the Volterra-Green method will not be used since this method reduces the solution of the problem under consideration to an integral equation. Attempts to obtain a solution to this equation appear to be hopeless. In order to find the unsteady loading function $\Psi$ it will first be necessary to determine the steady loading function, $\Psi^{*}=\Psi^{*}\left(x_{1}, y_{1}, z_{1}\right)$ characterized by

$$
-\frac{\partial^{2} \Psi^{*}}{\partial x_{1}^{2}}+\frac{\partial^{2} \Psi^{*}}{\partial y_{1}^{2}}+\frac{\partial^{2} \Psi^{*}}{\partial z_{1}^{2}}=0
$$

and

$$
\begin{gathered}
\left.\frac{\partial \Psi^{*}}{\partial z_{1}}\right|_{z_{1}=0}=\frac{e^{-\beta x_{1}}}{\Lambda \mu} f\left(x_{1}, y_{1}\right), \quad 0 \leq x_{1} \leq \Lambda h, \quad \Lambda \mu l \geq y_{1} \geq 0 \\
\Psi^{*}\left(\Gamma_{1}, \Gamma_{2}\right)=0 ; \quad \frac{\partial}{\partial \nu} \Psi^{*}\left(\Gamma_{1}, \Gamma_{2}\right)=0 .
\end{gathered}
$$

After $\Psi^{*}\left(x_{1}, y_{1}, z_{1}\right)$ the so called steady loading function is determined, then by use of the operational calculus the unsteady loading function $\Psi\left(x_{1}, y_{1}, z_{1}\right)$ will be expressed in an integral form, whose integrand contains the steady loading function $\Psi^{*}\left(x_{1}, y_{1}, z_{1}\right)$. This is the main object of this study.

4. Solution for the steady loading function. Evidently, the solution for steady loading function, $\Psi^{*}\left(x_{1}, y_{1}, z_{1}\right)$ will be determined as soon as a function $\Psi_{0}^{*}=\Psi^{*}\left(x_{1}, y_{1}, z_{1}\right)$ is determined such that the partial differential equation

$$
-\frac{\partial^{2} \Psi_{0}^{*}}{\partial x_{1}^{2}}+\frac{\partial^{2} \Psi_{0}^{*}}{\partial y_{1}^{2}}+\frac{\partial^{2} \Psi_{0}^{*}}{\partial z_{1}^{2}}=0
$$

subjected to the boundary conditions

$$
\begin{gathered}
\left.\frac{\partial \Psi_{0}^{*}}{\partial z_{1}}\right|_{s_{1}=0}=1, \quad 0 \leq x_{1} \leq \Lambda h, \quad \Lambda \mu h \geq y_{1} \geq 0 \\
\Psi_{0}^{*}\left(\Gamma_{1}, \Gamma_{2}\right)=0 \\
\frac{\partial}{\partial \nu} \Psi_{0}^{*}\left(\Gamma_{1}, \Gamma_{2}\right)=0
\end{gathered}
$$

is solved. 
Then the steady loading function, satisfying (23), (24) and (25) is given by

$$
\left.\Psi^{*}\right|_{z_{1}-0}=\left.\frac{1}{\Lambda \mu} \int_{0}^{x_{1}} \Psi_{0}^{*}\left(\xi_{1}, y_{1}, z_{1}\right)\right|_{z_{1}=0} \frac{\partial}{\partial \xi_{1}}\left[e^{-\beta\left(x_{1}-\xi_{1}\right)} f\left(x_{1}-\xi_{1}, y_{1}\right)\right] d \xi_{1}
$$

Note that (24) can be written as

$$
\left.\frac{\partial \Psi^{*}}{\partial z_{1}}\right|_{z_{1}=0}=\frac{1}{\Lambda \mu} \int_{0}^{x_{1}} \frac{\partial}{\partial x_{1}}\left[e^{-\beta x_{1}} f\left(x_{1}, y_{1}\right)\right] d x_{1} .
$$

Integrating (30) by parts, then

$$
\begin{aligned}
\left.\Psi^{*}\right|_{z_{1}=0}=\frac{1}{\Lambda \mu}\{- & \left.\left.\Psi_{0}^{*}\left(\xi_{1}, y_{1}, z_{1}\right)\right|_{z_{1}=0}\left[e^{-\beta\left(x_{1}-\xi_{1}\right)} f\left(x_{1}-\xi_{1}, y_{1}\right)\right]\right|_{0} ^{x_{1}} \\
& \left.+\left.\int_{0}^{x_{1}}\left[\frac{\partial}{\partial \xi_{1}} \Psi_{0}^{*}\left(\xi_{1}, y_{1}, z_{1}\right)\right]\right|_{z_{1}=0} e^{-\beta\left(x_{1}-\xi_{1}\right)} f\left(x_{1}-\xi_{1}, y_{1}\right) d \xi_{1}\right\} .
\end{aligned}
$$

Or, since the free term vanishes by virtue of boundaries, then

$$
\left.\Psi^{*}\right|_{z_{1}=0}=\left.\frac{1}{\Lambda \mu} \int_{0}^{x_{1}}\left[\frac{\partial}{\partial \xi_{1}} \Psi_{0}^{*}\left(\xi_{1}, y_{1}, z_{1}\right)\right]\right|_{z_{1}=0} e^{-\beta\left(x_{1}-\xi_{1}\right)} f\left(x_{1}-\xi_{1}, y_{1}\right) d \xi_{1} .
$$

For a wing of rectangular from Galin and Kovaleva adopted Busemann's solution [1. eq. 15] for which a derivative $\partial /\left.\partial x_{1} \Psi_{0}^{*}\left(x_{1}, y_{1}, z_{1}\right)\right|_{z_{1}=0}$ exists. As stated by Busemann, we have

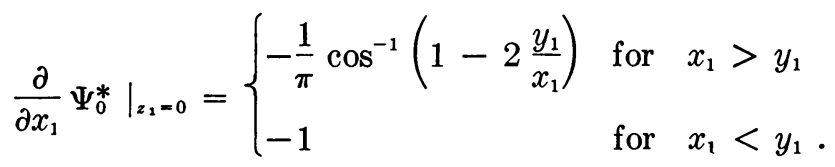

Hence, by the use of (34) the steady loading function can be obtained by direct quadrature of (33).

5. Relation between steady and unsteady loading functions. Define

$$
\begin{gathered}
F\left(\alpha, y_{1}, z_{1}\right)=\alpha \int_{0}^{\infty} e^{-\alpha x_{1}} \Psi\left(x_{1}, y_{1}, z_{1}\right) d x_{1} \\
F^{*}\left(\alpha, y_{1}, z_{1}\right)=\alpha \int_{0}^{\infty} e^{-\alpha x_{1}} \Psi^{*}\left(x_{1}, y_{1}, z_{1}\right) d x_{1} .
\end{gathered}
$$

Integrating (35) by parts it follows that

$$
F\left(\alpha, y_{1}, z_{1}\right)=\alpha^{-1} \int_{0}^{\infty} e^{-\alpha x_{1}} \frac{\partial^{2}}{\partial x_{1}^{2}} \Psi\left(x_{1}, y_{1}, z_{1}\right) d x_{1} .
$$

Then (19) and (37) lead to

$$
\frac{\partial^{2}}{\partial y_{1}^{2}} F\left(\alpha, y_{1}, z_{1}\right)+\frac{\partial^{2}}{\partial z_{1}^{2}} F\left(\alpha, y_{1}, z_{1}\right)-\left(\alpha^{2}-1\right) F\left(\alpha, y_{1}, z_{1}\right)=\underline{0} .
$$

Using the same technique (23) and (36) lead to

$$
\frac{\partial^{2}}{\partial y_{1}^{2}} F^{*}\left(\alpha, y_{1}, z_{1}\right)+\frac{\partial^{2}}{\partial z_{1}^{2}} F^{*}\left(\alpha, y_{1}, z_{1}\right)-\alpha^{2} F^{*}\left(\alpha, y_{1}, z_{1}\right)=0
$$


Evidently, (38) and (39) imply

$$
F\left(\alpha, y_{1}, z_{1}\right)=F^{*}\left[\left(\alpha^{2}-1\right)^{1 / 2}, y_{1}, z_{1}\right]
$$

Then

$$
\left.F\left(\alpha, y_{1}, z_{1}\right)\right|_{z_{2}=0}=\left.F^{*}\left[\left(\alpha^{2}-1\right)^{1 / 2}, y_{1}, z_{1}\right]\right|_{z_{1}=0} .
$$

It should be pointed out that (41) represents the functional relation between steady and unsteady loading function by means of operational calculus in the sense of (35), (36). However, from (24) the following relation holds

$$
\frac{1}{\Lambda \mu} e^{-\beta\left(x_{1}-\xi_{1}\right)} f\left(x_{1}-\xi_{1}, y_{1}\right)=\left.\frac{\partial}{\partial z_{1}} \Psi^{*}\left(x_{1}-\xi_{1}, y_{1}, z_{1}\right)\right|_{z_{1}=0} .
$$

Therefore, (33) and (42) lead to

$$
\left.\Psi^{*}\right|_{z_{1}=0}=\left.\left.\int_{0}^{x_{1}}\left[\frac{\partial}{\partial z_{1}} \Psi^{*}\left(x_{1}-\xi_{1}, y_{1}, z_{1}\right)\right]\right|_{z_{1}=0}\left[\frac{\partial}{\partial \xi_{1}} \Psi_{0}^{*}\left(\xi_{1}, y_{1}, z_{1}\right)\right]\right|_{z_{1}=0} d \xi_{1} \text {. }
$$

Denote

$$
\begin{aligned}
\left.\left.\mathscr{L}\left\{\frac{\partial}{\partial \xi_{1}} \Psi_{0}^{*}\left(\xi_{1}, y_{1}, z_{1}\right)\right\}\right|_{z_{1}=0} \equiv T\left(\alpha, y_{1}, z_{1}\right)\right|_{z_{1}=0} & \\
& =\left.\alpha \int_{0}^{\infty} e^{-\alpha \xi_{1}} \frac{\partial}{\partial \xi_{1}} \Psi_{0}^{*}\left(\xi_{1}, y_{1}, z_{1}\right)\right|_{z_{1}=0} d \xi_{1} .
\end{aligned}
$$

Evidently, from (36) it follows that

$$
\begin{aligned}
\left.\mathscr{L}\left\{\frac{\partial}{\partial z_{1}} \Psi^{*}\left(x_{1}, y_{1}, z_{1}\right)\right\}\right|_{z_{1}=0} \equiv \frac{\partial}{\partial z_{1}} F^{*}(\alpha & \left., y_{1}, z_{1}\right)\left.\right|_{z_{1}=0} \\
& =\left.\alpha \int_{0}^{\infty} e^{-\alpha x_{1}} \frac{\partial}{\partial z_{1}} \Psi^{*}\left(x_{1}, y_{1}, z_{1}\right)\right|_{z_{1}=0} d \xi_{1} .
\end{aligned}
$$

Then (43) by virtue of (44) and (45) represents the Faltung integral; namely, (43) can be written as

$$
\begin{aligned}
F^{*} & {\left.\left[\left(\alpha^{2}-1\right)^{1 / 2}, y_{1}, z_{1}\right]\right|_{z_{1}=0} } \\
& =\left.\left.\left(\alpha^{2}-1\right)^{-\frac{1}{2}}\left[\frac{\partial}{\partial z_{1}} F^{*}\left[\left(\alpha^{2}-1\right)^{1 / 2}, y_{1}, z_{1}\right)\right]\right|_{z_{1}=0} T\left[\left(\alpha^{2}-1\right)^{1 / 2}, y_{1}, z_{1}\right]\right|_{z_{1}=0} .
\end{aligned}
$$

Then from (41) and (46) it follows that

$$
\begin{aligned}
& \left.F\left(\alpha, y_{1}, z_{1}\right)\right|_{z_{1}=0} \\
& \quad=\left.\left.\left(\alpha^{2}-1\right)^{-1 / 2}\left[\frac{\partial}{\partial z_{1}} F^{*}\left[\left(\alpha^{2}-1\right)^{1 / 2}, y_{1}, z_{1}\right]\right]\right|_{z_{2}=0} T\left[\left(\alpha^{2}-1\right)^{1 / 2}, y_{1}, z_{1}\right]\right|_{z_{1}=0} .
\end{aligned}
$$

But

$$
\left.\frac{\partial}{\partial z_{1}} F\left(\alpha, y_{1}, z_{1}\right)\right|_{z_{1}=0}=\frac{\alpha}{\Lambda \mu} \int_{0}^{\infty} e^{-\alpha x_{1}}\left[e^{-\beta x_{1}} f\left(x_{1}, y_{1}\right)\right] d x_{1} .
$$

Therefore, (41) and (47) lead to

$$
\left.F\left(\alpha, y_{1}, z_{1}\right)\right|_{z_{1}=0}=\left.\left.\left(\alpha^{2}-1\right)^{-1 / 2}\left[\frac{\partial}{\partial z_{1}} F\left(\alpha, y_{1}, z_{1}\right)\right]\right|_{z_{1}=0} T\left[\left(\alpha^{2}-1\right)^{1 / 2}, y_{1}, z_{1}\right]\right|_{z_{1}=0}
$$


Clearly, (49) can be written as

$\left.F\left(\alpha, y_{1}, z_{1}\right)\right|_{z_{1}=0}$

$$
=\left.\left.\left[\alpha^{-1} \frac{\partial}{\partial z_{1}} F\left(\alpha, y_{1}, z_{1}\right)\right]\right|_{z_{1}=0}\left[\alpha\left(\alpha^{2}-1\right)^{-1 / 2} T\left[\left(\alpha^{2}-1\right)^{1 / 2}, y_{1}, z_{1}\right]\right]\right|_{z_{1}=0} .
$$

Moreover, the term in the first bracket on the right side can be modified. Firstly, note that $\partial / \partial z_{1} F\left(\alpha, y_{1}, z_{1}\right)$ is of exponential order. Then

$$
\mathscr{L}\left\{\frac{1}{\Lambda \mu} \int_{0}^{x_{1}} e^{-\beta \eta} f\left(\eta, y_{1}\right) d \eta\right\}=\left.\alpha^{-1} \frac{\partial}{\partial z_{1}} F\left(\alpha, y_{1}, z_{1}\right)\right|_{z_{1}=0} .
$$

However, the term in the second bracket of (50) must be developed. Denote

$$
T\left(\alpha, y_{1}, z_{1}\right)=\mathscr{L}\left\{f^{*}(t)\right\}
$$

where

$$
\mathscr{L}\left\{f^{*}(t)\right\}=\alpha \int_{0}^{\infty} e^{-\alpha t} f^{*}(t) d t
$$

But from a table of Laplace Transforms

$$
\mathscr{L}^{-1}\left\{\left[e^{-k\left(\alpha^{2}-a^{2}\right)^{1 / 2}}-e^{-\alpha k}\right] \alpha\right\}= \begin{cases}0, & 0<t<k \\ a k\left(t^{2}-k^{2}\right)^{-1 / 2} I_{1}\left[a\left(t^{2}-k^{2}\right)^{1 / 2}\right], & t>k\end{cases}
$$

where $I_{1}$, is a modified Bessel function of the first order. Putting $a=1$ and $k=u$ in (54) it follows after taking the Laplace Transform that

$$
\left(e^{-\left(\alpha^{2}-1\right)^{1 / 2} u}-e^{-\alpha u}\right)=\alpha^{-1} \mathcal{L}\left\{u\left(t^{2}-u^{2}\right)^{-1 / 2} I_{1}\left[\left(t^{2}-u^{2}\right)^{1 / 2}\right]\right\} .
$$

Evidently,

$$
\left(e^{-\left(\alpha^{2}-1\right)^{1 / 2} u}-e^{-\alpha u}\right)=\int_{0}^{\infty} e^{-\alpha t} u\left(t^{2}-u^{2}\right)^{-1 / 2} I_{1}\left[\left(t^{2}-u^{2}\right)^{1 / 2}\right] d t
$$

with the condition that the integrand is zero for $t<u$. Hence,

$$
\left(e^{-\left(\alpha^{2}-1\right)^{1 / 2} u}-e^{-\alpha u}\right) f^{*}(u)=\int_{0}^{\infty} e^{-\alpha t} u\left(t^{2}-u^{2}\right)^{-1 / 2} f^{*}(u) I_{1}\left[\left(t^{2}-u^{2}\right)^{1 / 2}\right] d t .
$$

Integrating (57) from 0 to $\infty$ with respect to $u$, it follows that

$$
\int_{0}^{\infty}\left(e^{-\left(\alpha^{2}-1\right)^{1 / 2} u}-e^{-\alpha u}\right) f^{*}(u) d u=\int_{0}^{\infty} \int_{0}^{\infty} e^{-\alpha t} u\left(t^{2}-u^{2}\right)^{-1 / 2} f^{*}(u) I_{1}\left[\left(t^{2}-u^{2}\right)^{1 / 2}\right] d t d u .
$$

Denote the left side of (58) by $L$ and right side by $R$, then in accordance with (52) it follows that:

$$
\int_{0}^{\infty} e^{-\alpha u} f^{*}(u) d u=\left.\alpha^{-1} T^{\prime}\left(\alpha, y_{1}, z_{1}\right)\right|_{z_{1}=0}
$$

and

$$
\int_{0}^{\infty} e^{-\left(\alpha^{2}-1\right)^{1 / 2} u} f^{*}(u) d u=\left.\left(\alpha^{2}-1\right)^{-1 / 2} T\left[\left(\alpha^{2}-1\right)^{1 / 2}, y_{1}, z_{1}\right]\right|_{z_{1}=0}
$$


Therefore,

$$
L=\left.\left(\alpha^{2}-1\right)^{-1 / 2} T\left[\left(\alpha^{2}-1\right)^{1 / 2}, y_{1}, z_{1}\right]\right|_{z_{1}=0}-\left.\alpha^{-1} T\left(\alpha, y_{1}, z_{1}\right)\right|_{z_{1}=0} .
$$

However,

$$
R=\int_{0}^{\infty} e^{-\alpha t} d t \int_{0}^{\infty} u\left(t^{2}-u^{2}\right)^{-1 / 2} f^{*}(u) I_{1}\left[\left(t^{2}-u^{2}\right)^{1 / 2}\right] d u
$$

But as the integrand is zero when $t<u$, we have

$$
R=\int_{0}^{\infty} e^{-\alpha t}\left[\int_{0}^{t} u\left(t^{2}-u^{2}\right)^{-1 / 2} f^{*}(u) I_{1}\left[\left(t^{2}-u^{2}\right)^{1 / 2}\right] d u\right] d t .
$$

Evidently,

$$
R=\alpha^{-1} \mathfrak{L}\left\{\int_{0}^{t} u\left(t^{2}-u^{2}\right)^{-1 / 2} f^{*}(u) I_{1}\left[\left(t^{2}-u^{2}\right)^{1 / 2}\right] d u\right\}
$$

Let $t^{2}-u^{2}=\tau^{2}$

Then

$$
u=\left(t^{2}-\tau^{2}\right)^{1 / 2}
$$

and

$$
u\left(t^{2}-u^{2}\right)^{-1 / 2} d u=-d \tau
$$

Therefore,

$$
R=\alpha^{-1} \mathscr{L}\left\{\int_{0}^{t} I_{1}(\tau) f^{*}\left[\left(t^{2}-\tau^{2}\right)^{1 / 2}\right] d \tau\right\}
$$

Evidently, (61) and (66) lead to

$$
\begin{aligned}
\left.\left(\alpha^{2}-1\right)^{-1 / 2} T\left[\left(\alpha^{2}-1\right)^{1 / 2}, y_{1}, z_{1}\right]\right|_{z_{1}=0}-\left.\alpha^{-1} T\left(\alpha, y_{1}, z_{1}\right)\right|_{z_{1}=0} & \\
& =\alpha^{-1} \&\left\{\int_{0}^{t} I_{1}(\tau) f^{*}\left[\left(t^{2}-\tau^{2}\right)^{1 / 2}\right] d \tau\right\},
\end{aligned}
$$

or

$$
\begin{aligned}
\left.\alpha\left(\alpha^{2}-1\right)^{-1 / 2} T\left[\left(\alpha^{2}-1\right)^{1 / 2}, y_{1}, z_{1}\right]\right|_{z_{1}=0}=\left.T\left(\alpha, y_{1}, z_{1}\right)\right|_{z_{1}=0} & \\
& +\mathscr{L}\left\{\int_{0}^{t} I_{1}(\tau) f^{*}\left[\left(t^{2}-\tau^{2}\right)^{1 / 2}\right] d \tau\right\} .
\end{aligned}
$$

Therefore,

$$
\left.\alpha\left(\alpha^{2}-1\right)^{-1 / 2} T\left[\left(\alpha^{2}-1\right)^{1 / 2}, y_{1}, z_{1}\right]\right|_{z_{1}=0}=\mathcal{L}\left\{f^{*}(t)+\int_{0}^{t} I_{1}(\tau) f^{*}\left[\left(t^{2}-\tau^{2}\right)^{1 / 2}\right] d \tau\right\}
$$

Eq. (67) by virtue of (44) can be written as

$$
\begin{aligned}
\left.\alpha\left(\alpha^{2}-1\right)^{-1 / 2} T\left[\left(\alpha^{2}-1\right)^{1 / 2}, y_{1}, z_{1}\right]\right|_{z_{1}=0}=\mathscr{L}\left\{\left.\frac{\partial}{\partial \xi_{1}} \Psi_{0}^{*}\left(\xi_{1}, y_{1}, z_{1}\right)\right|_{z_{1}=0}\right. \\
\\
\left.+\left.\int_{0}^{\xi_{1}} I_{1}\left(\xi_{2}\right) \frac{\partial}{\partial \xi_{2}} \Psi_{0}^{*}\left[\left(\xi_{1}^{2}-\xi_{2}^{2}\right)^{1 / 2}, y_{1}, z_{1}\right]\right|_{z_{1}=0} d \xi_{2}\right\}
\end{aligned}
$$


Clearly, (50), (51), and (70) lead to

$$
\begin{aligned}
\left.F\left(\alpha, y_{1}, z_{1}\right)\right|_{z_{1}=0}=\mathscr{L}\left\{\frac{1}{\Lambda \mu} \int_{0}^{x_{2}}\right. & \left.e^{-\beta \eta} f\left(\eta, y_{1}\right) d \eta\right\} \mathscr{L}\left\{\left.\frac{\partial}{\partial \xi_{1}} \Psi_{0}^{*}\left(\xi_{1}, y_{1}, z_{1}\right)\right|_{z_{1}=0}\right. \\
& \left.+\left.\int_{0}^{\xi_{1}} I_{1}\left(\xi_{2}\right) \frac{\partial}{\partial \xi_{2}} \Psi_{0}^{*}\left[\left(\xi_{1}^{2}-\xi_{2}^{2}\right)^{1 / 2}, y_{1}, z_{1}\right]\right|_{z_{1}-0} d \xi_{2}\right\} .
\end{aligned}
$$

Therefore,

$$
\begin{aligned}
&\left.\mathscr{L}^{-1}\left\{F\left(\alpha, y_{1}, z_{1}\right)\right\}\right|_{2_{1}=0}=\left[\frac{1}{\Lambda \mu} \int_{0}^{x_{1}} e^{-\beta \eta} f\left(\eta, y_{1}\right) d \eta\right] *\left[\left.\frac{\partial}{\partial \xi_{1}} \Psi_{0}^{*}\left(\xi_{1}, y_{1}, z_{1}\right)\right|_{z_{1}=0}\right. \\
&\left.+\left.\int_{0}^{\xi_{1}} I_{1}\left(\xi_{2}\right) \frac{\partial}{\partial \xi_{2}} \Psi_{0}^{*}\left[\left(\xi_{1}^{2}-\xi_{2}^{2}\right)^{1 / 2}, y_{1}, z_{1}\right]\right|_{z_{1}=0} d \xi_{2}\right] .
\end{aligned}
$$

Finally,

$$
\begin{aligned}
\left.\Psi\left(x_{1}, y_{1}, z_{1}\right)\right|_{z_{1}=0}=\frac{1}{\Lambda \mu} & \int_{0}^{x_{1}}\left\{\int _ { 0 } ^ { x _ { 1 } - \xi _ { 1 } } e ^ { - \beta \eta } f ( \eta , y _ { 1 } ) d \eta \left[\left.\frac{\partial}{\partial \xi_{1}} \Psi_{0}^{*}\left(\xi_{1}, y_{1}, z_{1}\right)\right|_{z_{1}=0}\right.\right. \\
& \left.\left.+\left.\int_{0}^{\xi_{1}} I_{1}\left(\xi_{2}\right) \frac{\partial}{\partial \xi_{2}} \Psi_{0}^{*}\left[\left(\xi_{1}^{2}-\xi_{2}^{2}\right)^{1 / 2}, y_{1}, z_{1}\right]\right|_{z_{1}=0} d \xi_{2}\right]\right\} d \xi_{1} .
\end{aligned}
$$

Hence,

$$
\begin{aligned}
\left.\Psi(x, y, z)\right|_{z=0}=\frac{1}{\Lambda \mu} \int_{0}^{\Lambda x} & \left\{\int _ { 0 } ^ { \Lambda x - \xi _ { 2 } } e ^ { - \beta \eta } f ( \eta , \Lambda \mu y ) d \eta \left[\left.\frac{\partial}{\partial \xi_{1}} \Psi_{0}^{*}\left(\xi_{1}, \Lambda \mu y, \Lambda \mu z\right)\right|_{z=0}\right.\right. \\
& \left.\left.+\left.\int_{0}^{\xi_{1}} I_{1}\left(\xi_{2}\right) \frac{\partial}{\partial \xi_{2}} \Psi_{0}^{*}\left[\left(\xi_{1}^{2}-\xi_{2}^{2}\right)^{1 / 2}, \Lambda \mu y, \Lambda \mu z\right]\right|_{z=0} d \xi_{2}\right]\right\} d \xi_{1} .
\end{aligned}
$$

Eq. (74) represents the aims of this paper; in other words, the velocity potential function, (7) is completely defined. Hence, (12), which represents the lifting forces on rectangular wing, can be evaluated for practical needs with no difficulty by an engineer in the field.

6. Conclusion. The significance of the present analytic method in the theory of unsteady motion of the wing is evident. The solution resulting from this method is obtained in closed integral form, so that integrand contains steady solution. This method has great advantage over Volterra-Green's techniques, since Volterra-Green's technique always gives a solution in form of an integral equation which cannot be solved exactly. Moreover, the numerical evaluation of the solution can be made by every engineer working in this field, without any difficulties. This is of great importance in dealing with flutter problems in the design of transonic and supersonic aircraft.

Finally, it seems worthwhile to note that the same method can be applied to the wing of trapezoidal form by using a proper transformation which transforms a trapezoidal wing into a wing of rectangular form.

\section{BIBLIOGRAPHY}

1. A. Busemann, Infinitesimale kegelige Überschallströmung, Schriften, Deutsche Akademie der Luftfahrtforschung, 3, 1943

2. L. A. Galin, Wing of rectangular form in supersonic flow, P.M.M. 11, 165-475 (1947)

3. S. V. Falkovich, Lift force of a wing of finite span, P.M.M. 11, 171-177 (1947)

4. J. W. Miles, The potential theory of unsteady supersonic flow, Cambridge Univ. Press, (1959) 
5. J. W. Miles, A note on supersonic wing integral equations in unsteady flow, Aeron. Quarterly 3, 294 (1951-52)

6. M. M. Stanišic, On the acceleration potential function for nonsteady motion of a delta-wing in supersonic flight, Bulet. Inst. Polit. Iasi, (6) 10, 49-61 (1960)

7. M. M. Stanišic, On the solution of an integral equation appearing in delta-wing theory, ZAMM 40, $397-415$ (1960)

8. E. A. Krassilchtchikova, Method of integral equations in thin wing theory in compressible medium, 9th Intern. Congr. Appl. Mech., 3, 57-67 (1956)

9. M. M. Stanišic, A remark concerning the vibration of a delta-wing in supersonic flight, J. Aero. Sci. 26, 679-680 (1959)

10. V. A. Kovaleva, On the unsteady motion of wing of a rectangular form, P.M.M. 23, 1030-1041 (1959)

11. M. M. Stanišić, On the aerodynamic forces acting on a delta wing in supersonic flow with supersonic leading edges, ZAMM 41, 342-354 (1961) 